\title{
de los fenómenos de fluencia en sistemas posteriormente modificados
}

JOHANMES NOHAMASON

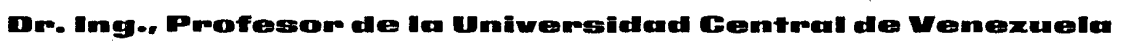

$400 \cdot 3$

La fluencia es la deformación plástica que tiene lugar en una pieza de hormigón, debido a las solicitaciones por compresión. Freyssinet fue el primero que desarrolló una teoría de la fluencia, de extraordinaria importancia en las estructuras de hormigón pretensado. Terzaghi ideó un modelo en el que se puede explicar muy bien el proceso físico que se realiza en el hormigón, el cual conduce a la deformación plástica.

En la siguiente exposición trataremos los efectos que experimenta la fluencia en el «mecanismo de las fuerzas internas» de una estructura cuyo sistema inicial ha sido modificado posteriormente. Un cambio posterior se realiza, por ejemplo, transformando vigas aisladas prefabricadas en una viga continua por medio de uniones adecuadas de los apoyos.

Otro cambio posterior de un sistema se efectuaría en el caso de un pórtico cuyo cabezal se pretensa, mientras que los montantes están parcialmente hormigonados. El hormigonado posterior, que trae consigo el aumento del momento de inercia de los montantes, representa una modificación de las características elásticas del sistema original.

Antes de entrar en la descripción matemática de los fenómenos mencionados, vamos a tratar los elementos fundamentales sobre los cuales el profesor Dischinger ha basado su teoría matemática de la fluencia en el hormigón.

El cuadro de las curvas estudiado por Glanville, demuestra la relación que existe entre las compresiones constantes y las deformaciones que se realizan en función del tiempo. Los ensayos fueron realizados utilizando un prisma de hormigón. Los dos fenómenos más importantes se observan directamente en el dibujo de las curvas:

1) Existe una relación lineal entre las deformaciones plásticas y las correspondientes tensiones, lo que nos dice que la ley Hook rige también en la zona de deformación plástica y, por lo tanto, la ley de superposición tiene validez para dichas deformaciones. La comparación entre las curvas de deformaciones-dilataciones, en el estado elástico plástico demuestra que la ley de Hook se cumple mejor en las deformaciones plásticas.

2) Las curvas demuestran también que los corrimientos plásticos son muy apreciables en los primeros cien días y disminuyen después de cuatro años. En general, se puede suponer que las deformaciones plásticas terminan a los cuatro años. La fluencia depende de las características del hormigón y otras circunstancias. 
Los factores que más influyen sobre la fluencia del hormigón son los siguientes:

a) En el hormigón pobre, las deformaciones plásticas son mayores que en el hormigón rico en cemento.

b) A mayor finura de la arena es la fluencia mayor.

c) La edad del hormigón en el momento de realizarse la carga tiene gran importancia; cuanto menor sea su «edad», mayor es la fluencia; el clima, especialmente la humedad del aire, tiene gran influencia sobre el comportamiento del hormigón bajo cargas permanentes.

Sin deformación elástica no aparece fluencia alguna; de ahí que sea lógico relacionar la deformación plástica con la elástica por medio de la expresión:

$$
\varphi=\frac{f_{t}}{\varepsilon}
$$

en la cual: $f_{t}$ es la deformación específica por fluencia después del tiempo $t$, y $\varepsilon$, la deformación específica elástica. El número absoluto $\phi$ lo podemos entonces considerar como un factor por el cual se tiene que multiplicar la deformación elástica para obtener la deformación plástica en un tiempo dado.

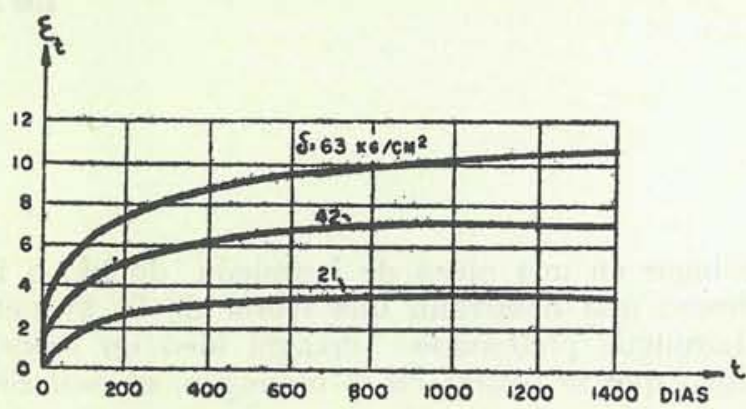

Curvas de deformacíon plástiea, según Glanville.

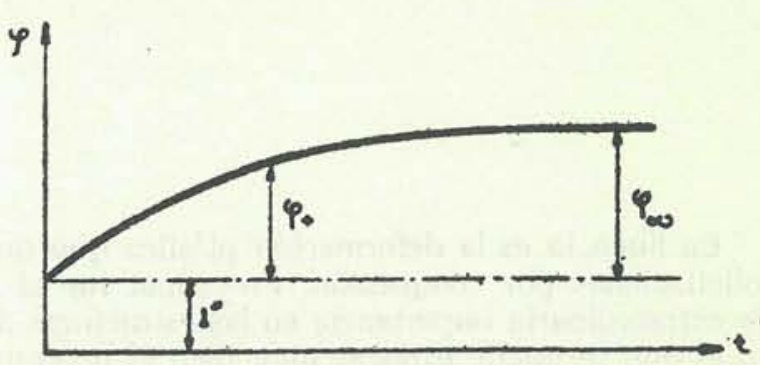

Función $\varphi(t)$, con $t$ variable dependiente del tiempo.

El factor $\varphi$ oscila entre 1 y 4, según las condiciones ya mencionadas. La curva de $\varphi$ se puede expresar analíticamente de manera aproximada por medio de la función (fig. 2):

$$
\varphi=\varphi_{\infty}\left(1-e^{-t}\right)
$$

\section{Ejemplo de un sistema «posteriormente modificado»}

Viga sobre tres apoyos, compuesta por dos vigas aisladas prefabricadas. Por medio de la unión esquemáticamente indicada en la figura 3 se transforman las dos vigas aisladas, después de su colocación, en una viga continua. La realización posterior de la unión significa que se ha efectuado la deformación elástica por peso propio (véase línea de puntos en la figura 4) cuando se unen las dos vigas.

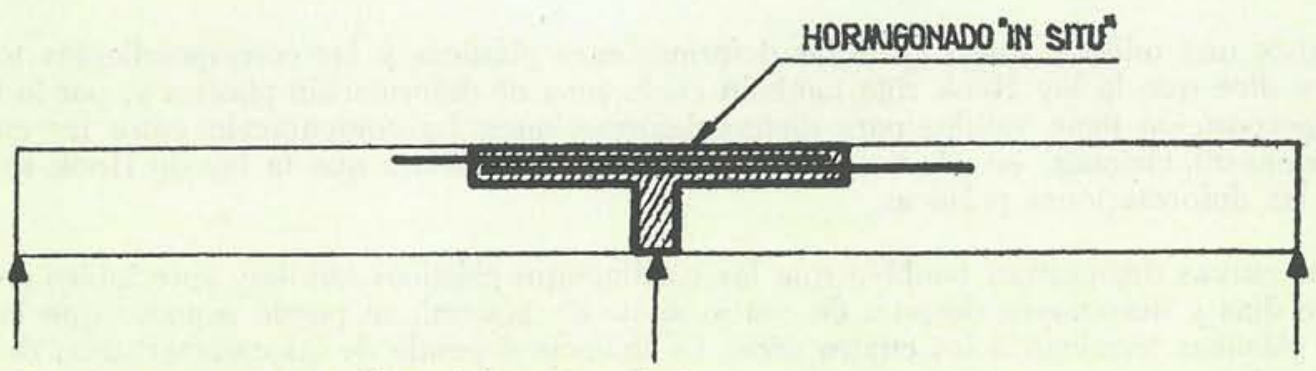

Viga sobre tres apoyos con unión posteriormente realizada. 


\section{Ecuación diferencial de plasticidad}

Para el cálculo del momento en el apoyo rige, en el estado elástico, la ecuación:

$$
\mathrm{X}_{1} \delta_{11}+\delta_{1,0}=0 \quad \mathrm{X}_{1}=-\frac{\delta_{10}}{\delta_{11}}
$$

donde: $\mathrm{X}_{1}$ es el momento desconocido en el apoyo; $\delta_{11}$, el ángulo de apertura efectuado por la hiperestática, y $\delta_{10}$, el ángulo de apertura efectuado por la carga exterior.

Como ya se ha dicho, el factor $\varphi$ relaciona las deformaciones plásticas con las deformaciones elásticas. Para la deformación total, después de un tiempo $t$, según la curva $\varphi$, se tiene (véase fig. 2) la expresión:

$$
\delta_{t}=\frac{\mathrm{M} \Delta \mathrm{X}}{\mathrm{EJ}} \cdot\left(1+\varphi_{t}\right)=\delta_{e l}(1-\varphi) \delta_{e l}=\text { Deformación elástica. }
$$

En esta expresión se ha despreciado la variación del módulo de elasticidad $\mathrm{E}$ durante el tiempo. $\mathrm{La}$ influencia de esta variación es muy pequeña. Al diferenciar respecto a $t$, nos da el aumento $d \delta_{t}$ de la deformación durante el intervalo de tiempo $d t$ :

$$
\frac{d \delta_{t}}{d t}=\delta_{e l} \frac{d \varphi}{d t}
$$

Después de estas notas preliminares podemos abordar el problema original. Durante el tiempo $t$ se efectúan deformaciones plásticas y elásticas. Una vez cerrada la junta, dichas deformaciones no pueden realizarse libremente $\mathrm{y}$ «despiertan», así, una hiperestática $\mathrm{X}_{t}$, a la cual damos el subíndice $t$, dependiendo la magnitud de este momento del tiempo que ha transcurrido a partir de haber entrado en función la junta. Para poder plantear la ecuación diferencial de $\mathrm{X}_{t}$ investigamos separadamente las deformaciones en el intervalo $d t$ en el sistema estáticamente determinado, solicitado por las cargas exteriores y la hiperestática $\mathrm{X}_{t}$ :

$$
\delta_{01} \rightarrow \delta_{01} d \varphi
$$

La expresión nos dice que la deformación elástica existente se aumenta en el intervalo $d t$, en $\delta_{01} d \varphi$, siendo la deformación total en dicho intervalo: $\delta_{01}+\delta_{01} d \varphi$.

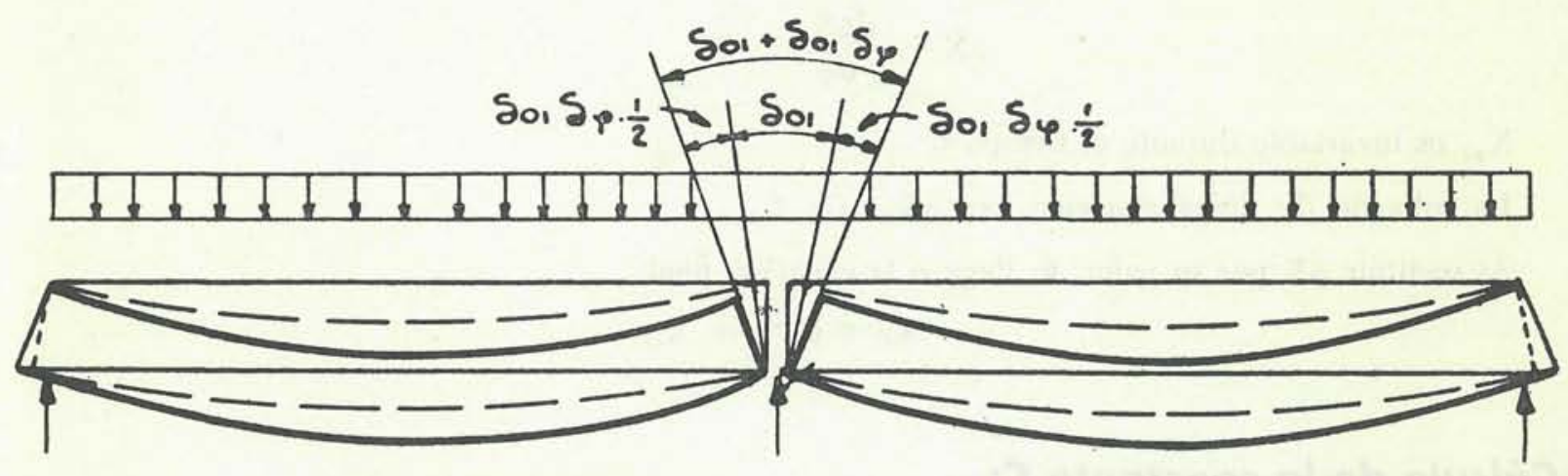

Deformaciones plásticas y elásticas en un sistema estáticamente determinado.

Después de un tiempo $d t$, la elástica original se ha transformado en la indicada (fig. 4) con trazo fuerte; a dicha elástica corresponde el nuevo ángulo de apertura $\delta_{01}+\delta_{01} d \varphi$ :

$$
\mathrm{X}_{t} \delta_{11} \rightarrow \mathrm{X}_{t} \delta_{11} d \varphi
$$

$\mathrm{X}_{t} \delta_{11}$ es la deformación efectuada en el sistema estáticamente determinado por la hiperestática $\mathrm{X}_{t}$, 
despertada después del tiempo $t$. Debido a la fluencia se aumenta la deformación de idéntica manera a la del caso anterior en $\mathrm{X}_{t} \delta_{11} \cdot d_{\varphi}$ :

$$
d \mathrm{X}_{t} \delta_{11}
$$

En el intervalo de tiempo $d t$, la hiperestática se incrementa en $d \mathrm{X}_{t}$, originándose la deformación $d \mathrm{X}_{t} \cdot \delta_{11}$. Dicho incremento $d \mathrm{X}_{t}$ en el intervalo $d t$ produce una deformación plástica cuyo valor es: $d \mathrm{X}_{t} \delta_{11} \cdot d \varphi$. Al ser esta expresión una diferencial de segundo orden, podemos despreciar la deformación plástica causada por $d \mathrm{X}_{t}$. Las condiciones geométricas deben estar satisfechas en un sistema con deformaciones plásticas de igual forma que si se tratara de un sistema puramente elástico.

En nuestro caso rige la condición de que todas las deformaciones (ángulos de giro) en el apoyo, deben ser iguales a cero en el intervalo $d t$ después del tiempo $t$. Llamemos $t$ al tiempo que ha pasado después de haber entrado en función la junta, y $\Delta$, a las deformaciones elásticas y plásticas en general. La condición $\Sigma \Delta=0$ durante $d t$, da la ecuación diferencial:

$$
\delta_{01} d_{\varphi}+\mathrm{X}_{t} \delta_{11} d_{\varphi}+d \mathrm{X}_{t} \delta_{11}=0
$$

y dividiendo por $\delta_{x 1} d_{\varphi}$ se obtiene:

$$
\frac{\delta_{01}}{\delta_{11}}+\mathbf{X}_{t}+\frac{d \mathbf{X}_{t}}{d \varphi}=0
$$

según la ecuación elástica:

$$
\frac{\delta_{01}}{\delta_{11}}=-\mathrm{X}_{e l} .
$$

$\mathrm{X}_{e l}$ es la hiperestática originada al ejecutar la viga como viga continua en forma convencional.

Introduciendo $\mathrm{X}_{e l}$ obtenemos:

$$
-\mathrm{X}_{e l}+\mathrm{X}_{t}+\frac{d \mathrm{X}_{t}}{d \varphi}=0
$$

Por medio del cambio

$$
-\mathrm{X}_{e l}+\mathrm{X}_{t}=\Delta \mathrm{X} \quad \text { y } \quad \frac{d \Delta \mathrm{X}}{d \varphi}=\frac{d \mathrm{X}_{t}}{d \varphi}
$$

se puede escribir la siguiente ecuación diferencial de primer orden:

$$
\Delta \mathrm{X}+\frac{d \Delta \mathrm{X}}{d \varphi}=0 \text {. }
$$

$\mathrm{X}_{c l}$ es invariable durante el tiempo $t$.

La solución de dicha ecuación es: $\Delta \mathrm{X}=\mathrm{C} e^{-\varphi}$

Al sustituir $\Delta \mathrm{X}$ por su valor, se llega a la ecuación final:

$$
\mathrm{X}_{t}=\mathrm{C}_{e}^{-\varphi}+\mathrm{X}_{e l}
$$

\section{Cálculo de la constante C:}

\section{Primera condición:}

Se cierra la junta antes de desencofrar la viga o se hormigona la viga «in situ».

Esto significa que la junta entra en función con el desencofrado de la viga. El momento de entrar en función la junta implica que $t=0$, obteniéndose las dos igualdades:

$$
\mathrm{X}_{t=0}=\mathrm{X}_{e l} \quad ; \quad \varphi_{t=0}=0
$$


Introduciendo estos valores en la ecuación diferencial obtenemos:

$$
\mathrm{X}_{e l}=\mathrm{C}+\mathrm{X}_{e l} \rightarrow \mathrm{C}=0 \quad ; \quad \mathrm{X}_{e l}=\mathrm{X}_{t}
$$

Este resultado demuestra que las deformaciones plásticas no modifican la magnitud de la hiperestática cuando la carga exterior es invariable y el sistema no cambia sus condiciones de apoyo.

\section{Segunda condición:}

\section{Cierre de la junta después de haber desencofrado las vigas:}

Este proceso corresponde, por supuesto, a la colocación de vigas prefabricadas y su unión posterior. Para $t=0$ quiere decir que inmediatamente después de haber cerrado la junta no existe momento en el apoyo, efecto del peso propio o por cualquier carga que actuaba antes de haber cerrado dicha junta:

$$
\mathrm{X}_{t=0}=0 ; \varphi_{t=0}=0
$$

Introduciendo estos valores en la solución general de la ecuación diferencial podemos calcular la constante $\mathrm{C}$ :

$$
0=\mathrm{C}+\mathrm{X}_{e l} \quad ; \mathrm{C}=-\mathrm{X}_{e l} \quad ; \quad \mathrm{X}_{t}=\mathrm{X}_{e l}\left(1-e^{-\varphi}\right)
$$

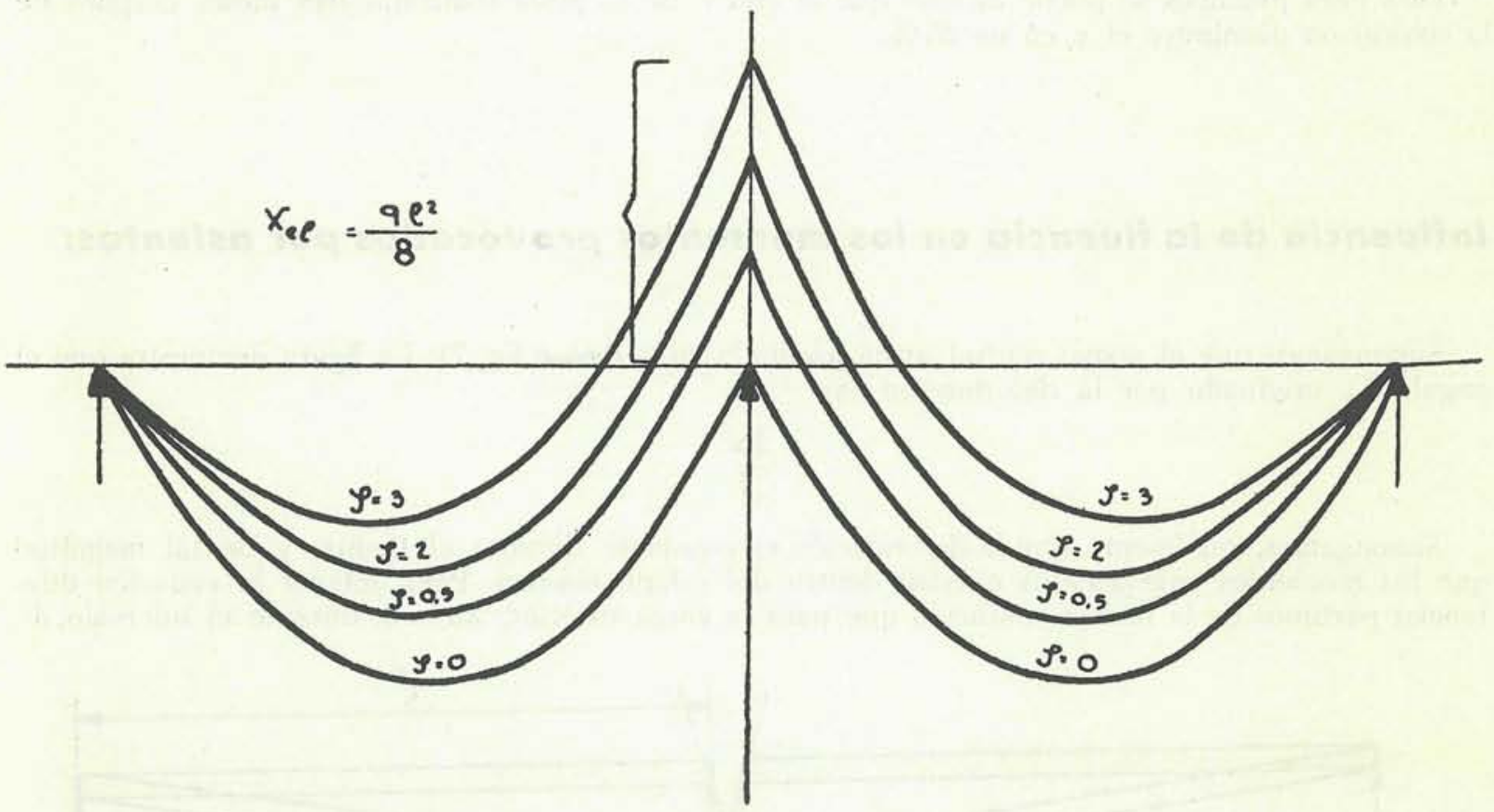

Aumento del momento en el apoyo, en función del tiempo.

Las curvas nos demuestran cómo se origina un momento negativo en el apoyo debido a la fluencia y el valor que corresponde al $95 \%$ del $\mathrm{X}_{e l}$ para un valor de $\varphi=3$. El lapso de tiempo transcurrido entre la colocación de las vigas y el cierre de la junta, tiene una influencia sobre la magnitud del valor $\varphi$. El cierre corresponde, como hemos visto, a la introducción de una nueva solicita- 
ción de la viga, pudiendo, por ello, aplicar con buena aproximación la relación encontrada por Withney para las curvas $\phi$ en los elementos cargados en tiempos diferentes. Dicha relación se puede expresar de la siguiente forma:

$$
\varphi_{t} \Delta_{t}=\varphi_{t}-\varphi_{t c}
$$

Esto nos dice que el número de fluencia $\varphi$ para una carga adicional, que actúa después de haber introducido la carga original, es igual al valor $\varphi_{t}$ para dicha carga menos el valor de fluencia $\varphi_{t e}$ que existió en el momento de la aplicación de la nueva carga (véase fig. 6). En nuestro caso, la carga original está representada por el peso propio, y la carga adicional, por el momento $\mathrm{X}_{t}$ «despertado» después del cierre de la junta.

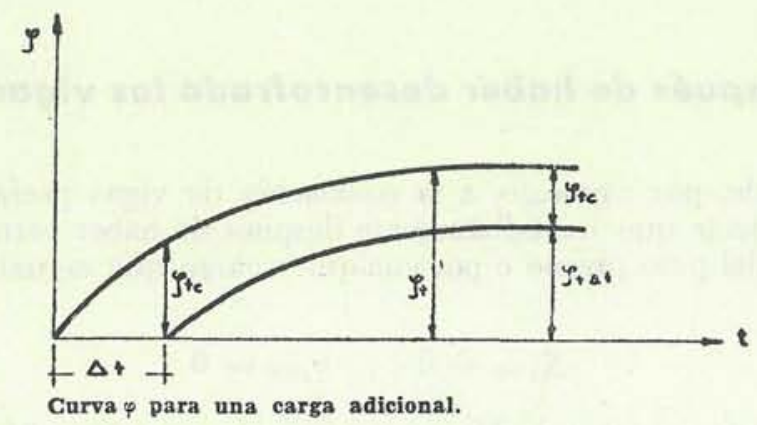

La aplicación de la relación de Withney es aproximada, debido a la variación del momento $\mathrm{X}_{t}$. Withney realizó sus experimentos con cargas constantes.

Para fines prácticos se puede estimar que el cierre de la junta realizado tres meses después de la colocación disminuye el $\varphi$ en un $25 \%$.

\section{Influencia de la fluencia en los momentos provocados por asientos:}

Supongamos que el apoyo central se ha asentado en $\Delta$ (véase fig. 7). La figura demuestra que el ángulo $\delta_{01}$ originado por la deformación es:

$$
\frac{2 \Delta}{l}
$$

Supongamos, igualmente, que la deformación es constante durante el tiempo y de tal magnitud que los momentos engendrados quedan dentro del estado elástico. Para obtener la ecuación diferencial partimos de la misma condición que para la carga exterior, $\Sigma \Delta=0$, durante el intervalo $d t$.

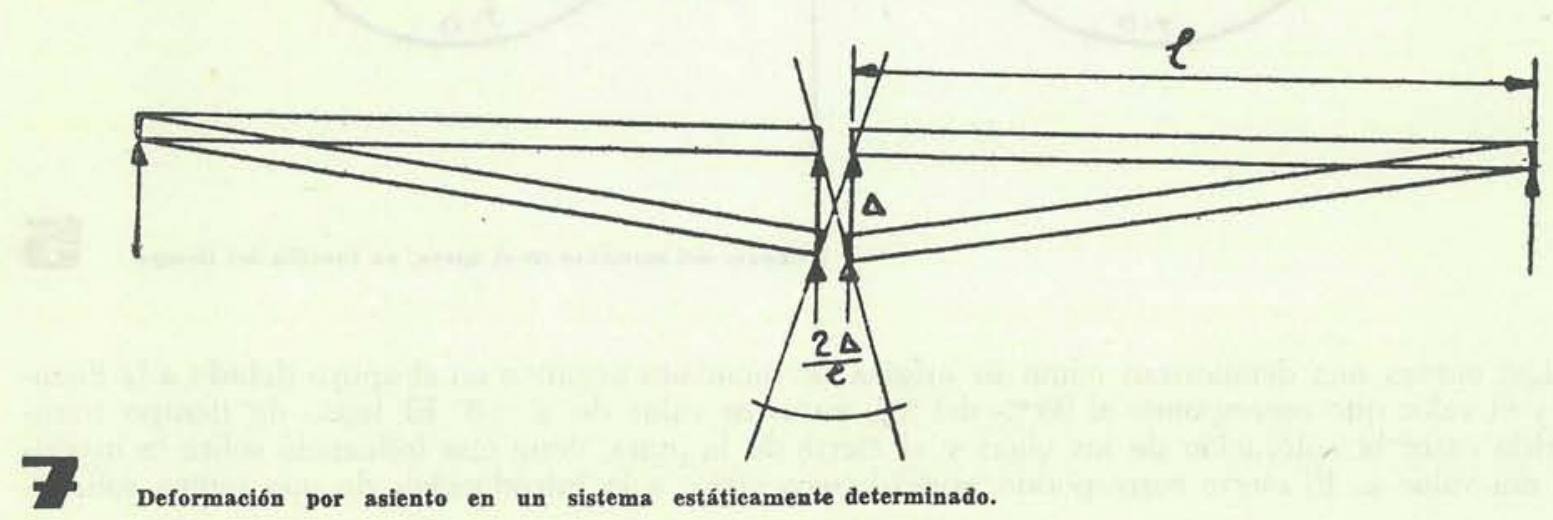


La ecuación derivada anteriormente fue:

$$
\delta_{01} d \varphi+\mathrm{X}_{t} \delta_{11} d \varphi+d \mathrm{X}_{t} \delta_{11}=0 .
$$

Siendo $\delta_{01}=2 \Delta / l$ invariable durante el tiempo en el caso de un asiento pequeño, en las condiciones indicadas anteriormente el término $\delta_{01} d \phi$ desaparece en la ecuación diferencial, la cual determina el cambio de la deformación en el intervalo $d t$.

Se obtiene así la ecuación diferencial más sencilla:

$$
\mathrm{X}_{t} \delta_{11} d_{\varphi}+d \mathrm{X}_{t} \delta_{11}=0 \quad ; \quad \mathrm{X}_{t}+\frac{d \mathrm{X}_{t}}{d \varphi}=0
$$

La solución es: $\mathrm{X}_{t}=\mathrm{C} e^{-\varphi}$

Para calcular la constante investigamos la condición inicial para $t=0$. Inmediatamente después de haberse realizado la deformación, $X_{t}$ es el momento engendrado por la deformación en el sistema elástico. En el caso de una viga sobre tres apoyos con luces iguales, este momento sería:

$$
3 \mathrm{EJ} \frac{\Delta}{l^{2}}
$$

para

$$
\varphi_{t=0}=0 \quad \mathrm{y}, \quad \mathrm{X}_{t=0}=\mathrm{X}_{e l} \quad \rightarrow \quad \mathrm{C}=\mathrm{X}_{e l} \quad \mathrm{y}, \quad \mathrm{X}_{t}=\mathrm{X}_{e l} e^{-\varphi}
$$

El momento en el apoyo por asiento disminuye con el tiempo hasta alcanzar, después de cuatro años, y con $\varphi=3$, un valor igual al $5 \%$ del valor original. Este fenómeno, por vez primera descubierto por Dischinger, tiene una gran importancia en la ejecución de obras. Las más importantes consecuencias son las siguientes:

1. Un asiento efectuado inmediatamente después del desencofrado es mucho menos peligroso de lo que se cree generalmente, reduciéndose la influencia desfavorable rápidamente en el hormigón de poca edad.

2. La introducción de momentos artificiales por deformación es prácticamente inefectiva. Al introducir, por ejemplo, una compresión por asiento en una placa de hormigón unido a una viga en I de acero, dicha compresión desaparece casi por completo.

\section{Influencia del pretensado al realizar un "cambio posterior del sistema»}

En el pórtico empotrado (véase fig. 8), la viga está pretensada y, posteriormente, se realiza la unión en las juntas $n-n$ con los soportes. Se han realizado estructuras de este tipo, y el procedimiento parece ser muy útil, ya que los soportes robustos absorben una gran parte del esfuerzo de pretensado, procedimiento que permite evitar la desventaja señalada.

Por medio de las ecuaciones de plasticidad podemos investigar también este fenómeno.

Por simetría, el sistema es dos veces hiperestático (véase fig. 9). El sistema de ecuaciones en estado elástico es el siguiente:

$$
\begin{array}{r}
\mathrm{X}_{1}^{e l} \delta_{11}+X_{2}^{e l} \delta_{12}+\delta_{10}=0 \\
\mathrm{X}_{1}{ }^{e l} \delta_{21}+\mathrm{X}_{2}^{e l} \delta_{22}+\delta_{20}=0
\end{array}
$$


Para las incógnitas $\mathrm{X}_{1}$ y $\mathrm{X}_{2}$ obtenemos los siguientes valores:

$$
\mathrm{X}_{1}{ }^{e l}=\frac{-\delta_{10} \delta_{22}+\delta_{12} \delta_{20}}{\mathrm{D}_{\mathrm{N}}}
$$

siendo:

$$
\begin{array}{r}
\mathrm{D}_{\mathrm{N}}=\delta_{11} \delta_{12}-\delta^{2}{ }_{12} \\
\mathrm{X}_{2}{ }^{e l}=\frac{-\delta_{11} \delta_{20}+\delta_{10} \delta_{21}}{\mathrm{D}_{\mathrm{N}}}
\end{array}
$$

Las consideraciones para obtener las ecuaciones diferenciales en el estado plástico son las mismas que las de la viga sobre tres apoyos. En el caso del pórtico, $\Sigma \Delta$ tiene que ser igual a cero en ambas secciones.
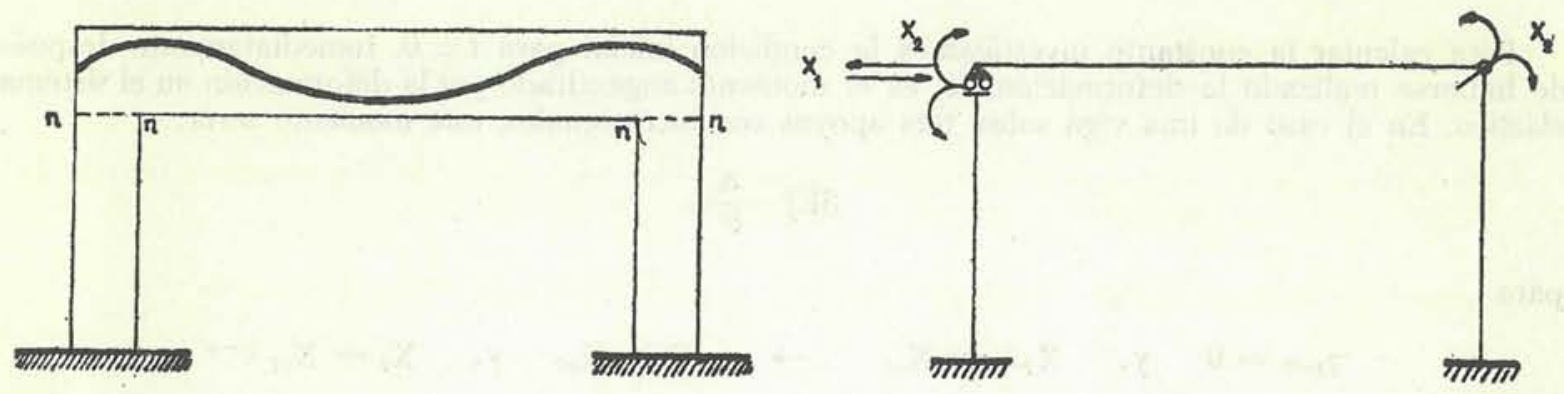

i. Pórtico con empotramiento en la viga pretensada.

Sistema inicial base para el cáleulo.

Estas dos condiciones conducen a un sistema de dos ecuaciones diferenciales:

$$
\begin{aligned}
& \mathrm{X}_{1}{ }^{t} \delta_{11} d \varphi+d \mathrm{X}_{1}{ }^{t} \delta_{11}+\mathrm{X}_{2}{ }^{t} \delta_{12} d \varphi+d \mathrm{X}_{2}{ }^{t} \delta_{12}+\delta_{10} d \varphi=0 \\
& \mathrm{X}_{1}{ }^{t} \delta_{12} d \varphi+d \mathrm{X}_{1}{ }^{t} \delta_{12}+\mathrm{X}_{2}{ }^{t} \delta_{22} d \varphi+d \mathrm{X}_{2}{ }^{t} \delta_{22}+\delta_{20} d \varphi=0
\end{aligned}
$$

Podemos transformar el sistema de dos ecuaciones diferenciales en dos ecuaciones diferenciales independientes multiplicando la ecuación [1] por $\frac{\delta_{22}}{D_{N}}$ y la ecuación [2] por $-\frac{\delta_{12}}{D_{N}}$

La operación conduce a las dos ecuaciones siguientes:

$$
\begin{aligned}
& \mathrm{X}_{1}{ }^{t} \frac{\delta_{11} \delta_{22} d \varphi}{\mathrm{D}_{\mathrm{N}}}+d \mathrm{X}_{1}{ }^{t} \frac{\delta_{11} \delta_{22}}{\mathrm{D}_{\mathrm{N}}}+\mathrm{X}_{2}{ }^{t} \frac{\delta_{12} \delta_{22}}{\mathrm{D}_{\mathrm{N}}} d \varphi+d \mathrm{X}_{2} \frac{{ }^{t} \delta_{12} \delta_{22}}{\mathrm{D}_{\mathrm{N}}}+\frac{\delta_{10} \delta_{22}}{\mathrm{D}_{\mathrm{N}}} d \varphi=0 \\
& -\mathrm{X}_{1}{ }^{t} \frac{\delta^{e}{ }_{12} d \varphi}{\mathrm{D}_{\mathrm{N}}}-d \mathrm{X}_{1}{ }^{t} \frac{\delta_{12}^{e}}{\mathrm{D}_{\mathrm{N}}}-\mathrm{X}_{2}{ }^{t} \frac{\delta_{22} \delta_{12}}{\mathrm{D}_{\mathrm{N}}} d \varphi-d \mathrm{X}_{2}{ }^{t} \frac{\delta_{22} \delta_{12}}{\mathrm{D}_{\mathrm{N}}}-\frac{\delta_{20} \delta_{12}}{\mathrm{D}_{\mathrm{N}}} d \varphi=0
\end{aligned}
$$

La suma de las dos ecuaciones, teniendo en cuenta que:

$$
-\mathrm{X}_{1}{ }^{e l}=\frac{\delta_{10} \delta_{22}-\delta_{20} \delta_{12}}{\mathrm{D}_{\mathrm{N}}}
$$

nos da la siguiente ecuación diferencial:

$$
\begin{aligned}
\mathrm{X}_{1}{ }^{t} d \varphi+d \mathrm{X}_{1}{ }^{t}-\mathrm{X}_{e l} d \varphi & =0 \\
-\mathrm{X}_{1}{ }^{e l}+\mathrm{X}_{1}{ }^{t}+\frac{d \mathrm{X}_{1}{ }^{t}}{d \varphi} & =0
\end{aligned}
$$

Esta ecuación es idéntica a la ecuación diferencial para el caso de una sola hiperestática, siendo su solución:

$$
\mathrm{X}_{1}^{t}=\mathrm{X}_{1}{ }^{e l}\left(1-e^{-\varphi}\right)
$$


Por un mecanismo exactamente igual al anterior, se obtiene una ecuación diferencial en $\mathrm{X}_{2}$ cuya solución será:

$$
\mathrm{X}_{2}{ }^{t}=\mathrm{X}_{2}{ }^{e l}\left(1-e^{-\varphi}\right)
$$

Así, vemos también que para un sistema con dos hiperestáticas rige, para el crecimiento de los momentos por fluencia, la misma ley $\left(1-e^{-\varphi}\right)$, que para el caso de una sola hiperestática.

Especialmente interesante es el hecho de que el crecimiento de una hiperestática es completamente independiente del aumento de la magnitud de la otra.

\section{Ejemplo numérico}

Supongamos que la junta $n-n$ se ha cerrado después de haberse realizado el pretensado en la viga. Sea el cable de forma perabólica y sometido a un esfuerzo de 80 toneladas.

El momento máximo debido al pretensado en el centro es de $0,35 \times 80=28$ metros.
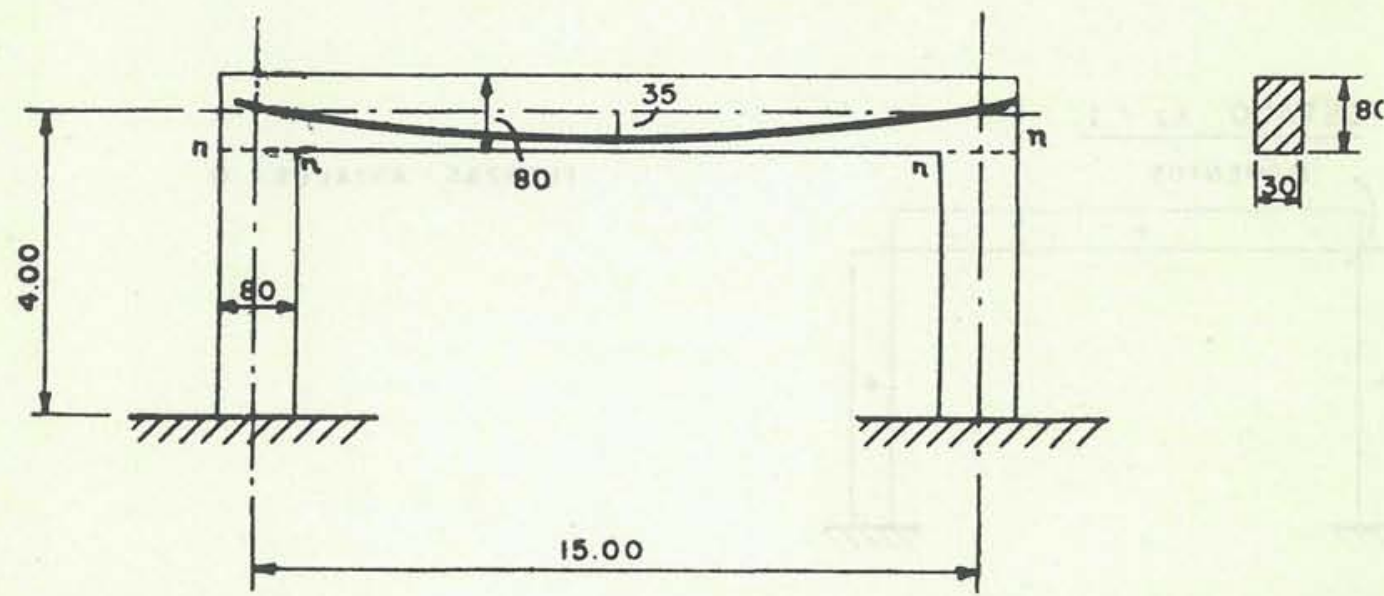

Dimensionado del pórtico empotrado.

Las características del sistema son:

$$
\frac{J}{A}=0,0535 \mathrm{~m}^{2} ; W=32.000 \mathrm{~cm}^{3} ; A=24.000 \mathrm{~cm}^{2} \text {. }
$$

Calculemos primeramente las hiperestáticas en el estado elástico, es decir, inmediatamente después del cierre de la junta:

Valores $\delta_{i k}$ :

$$
\mathrm{EJ}_{c} \delta_{i k}=\delta \mathrm{M}_{i} \mathrm{M}_{k} d s+\delta \mathrm{N}_{i} \mathrm{~N}_{k} \frac{\mathrm{J}}{\mathrm{A}} d s
$$

La influencia del esfuerzo cortante para las deformaciones $\delta_{i k}$ se ha despreciado. 


\section{ejemplo numérico}
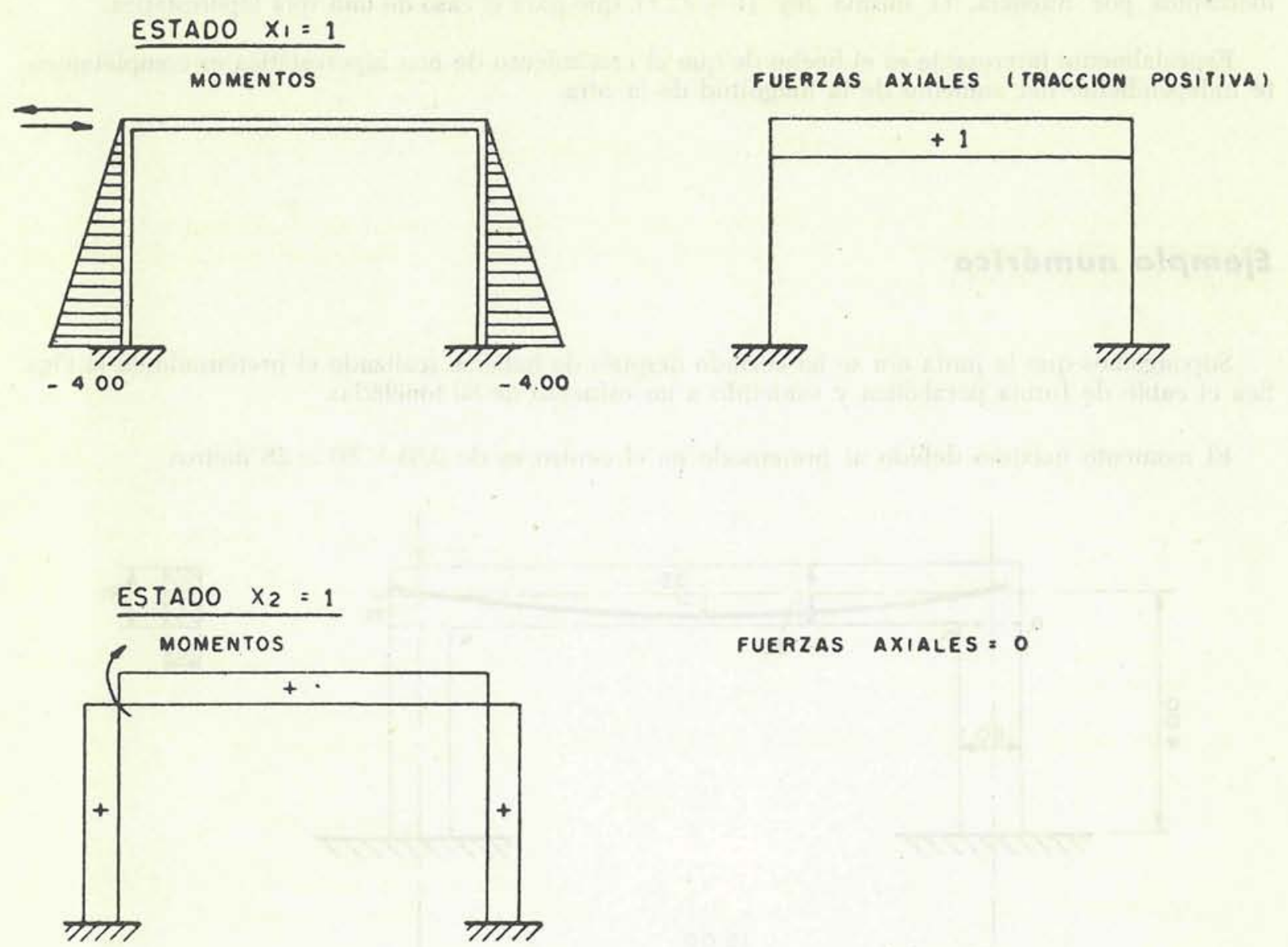

FUERZAS AXIALES $=0$
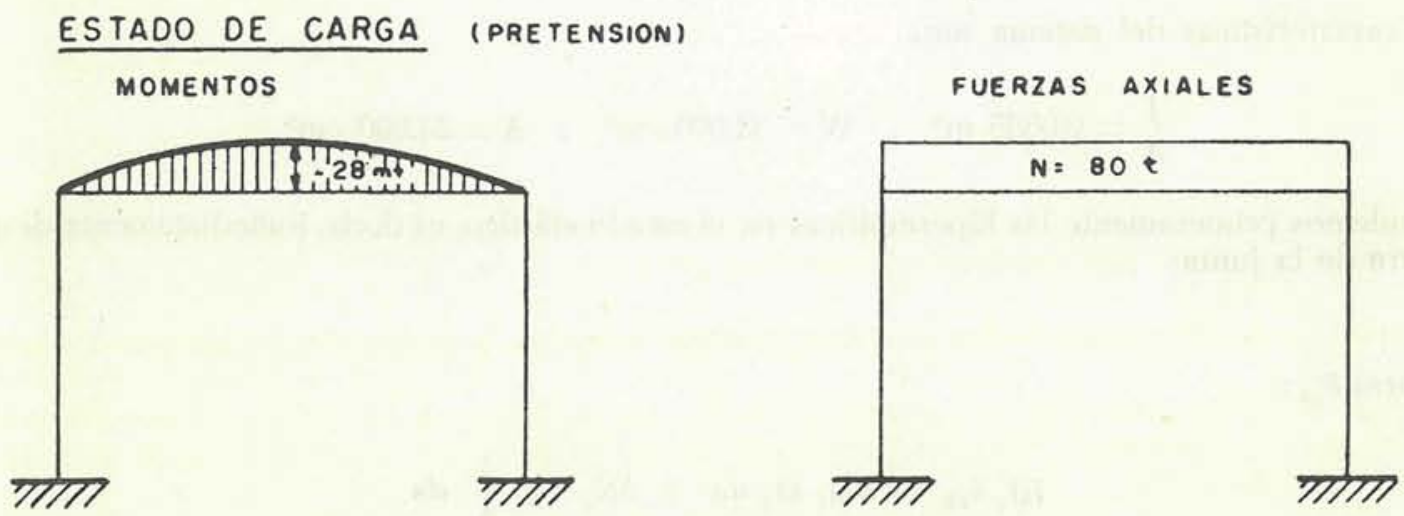
Los valores son:

$$
\begin{aligned}
& \mathrm{EJ}_{c} \delta_{11}=43,50 \\
& \mathrm{EJ}_{c} \delta_{22}=23,00 \\
& \mathrm{EJ}_{12}=16,00 \\
& \mathrm{EJ}_{10}=64,20 \\
& \mathrm{EJ}_{20}=280,00 \\
& \mathrm{X}_{1}{ }^{e l}=8,00 t . \quad ; \quad \mathrm{X}_{2}{ }^{e l}=17,85 .
\end{aligned}
$$

Estos valores $\mathrm{X}_{1}{ }^{e l}$ y $\mathrm{X}_{2}{ }^{e l}$ son las hiperestáticas que hubiesen existido en el sistema ejecutado en la manera usual y posteriormente pretensado. En nuestro caso, estos momentos entran parcialmente después de haber cerrado la junta.

La tabla demuestra el crecimiento de $\mathrm{X}_{1}{ }^{t}$ y $\mathrm{X}_{2}{ }^{t}$ para diferentes valores de $\varphi$ :

\begin{tabular}{lccccc}
\hline$\varphi$ & 1,0 & 2,0 & 2,5 & \multicolumn{1}{c}{3,0} & $\infty$ \\
$\mathrm{X}_{1}{ }^{t}$ & 5,06 & 6,90 & 7,37 & 7,62 & 8,00 \\
$\mathrm{X}_{2}{ }^{t}$ & 11,39 & 15,40 & 16,40 & 16,90 & 17,85 \\
\hline
\end{tabular}

Es interesante estudiar también la influencia de los momentos que han de considerarse debidos a la fluencia sobre la compresión que actúa en la sección central.

Inmediatamente después del cierre de la junta tenemos la siguiente compresión:

$$
\sigma=\frac{80.000}{2.400}+\frac{280.000}{32.000}=33+87=+120 \mathrm{~kg} / \mathrm{cm}^{2}
$$

Después de tres años, $\varphi \sim 3$ se tienen las siguientes fuerzas axiales y momentos:

$$
\begin{aligned}
& \mathrm{M}=+\mathrm{M}_{o}+\mathrm{M}_{2} \mathrm{X}_{2}=-28,0+16,90=-11,0 \mathrm{~m} \cdot \mathrm{t} . \\
& \mathrm{N}=\mathrm{N}_{\mathrm{o}}+\mathrm{N}_{1} \mathrm{X}_{1}=-80,0+7,62=-72,38 \mathrm{t} \text { (compresión). }
\end{aligned}
$$

Con estos valores obtenemos la compresión existente después de tres años en el corte central de la viga del pórtico:

$$
\sigma=\frac{72.380}{2.400}+\frac{1.110 .000}{32.000}=30,20+34,70=64,90 \mathrm{~kg} / \mathrm{cm}^{2} .
$$

El pretensado se ha reducido de un $46 \%$ en este caso. Una reducción tan fuerte de la compresión en la zona comprimida conduciría a considerables grietas.

Un aumento de la rigidez de los soportes reduciría aún más la acción del pretensado en la viga.

Después de haber visto que un pretensado de la viga preliminar a la transformación del sistema en pórtico por el cierre de la junta es poco efectivo, nos preguntamos si un sistema de este tipo es apto para el pretensado.

Para resolver el problema que introduce un estuerzo efectivo de pretensado en la viga existen dos posibilidades: 


\section{Acercamiento a un arco con tres articulaciones}

Una apropiada variación del momento de inercia conduce a un sistema cuyo diagrama de momentos se acerca al de un arco con tres articulaciones.

Acercándose, así, el comportamiento de la estructura hiperestática a un sistema estáticamente determinado, obtenemos pequeños momentos parásitos.

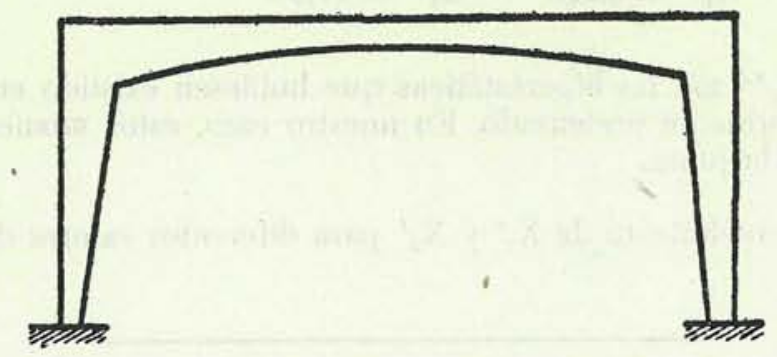

Variación del momento de inercia para reducir el momento parásito.

Esta idea fue aplicada en el proyecto del puente de acceso al helicoide, con objeto de reducir la pérdida de pretensado, debida a momentos parásitos, a solamente $9 \%$. A momentos parásitos menores corresponde, por supuesto, una deformación mayor, la cual se puede contrarrestar con una contra-flecha.

Una deformación de 3 a $4 \mathrm{~cm}$ no representa ningún problema en un puente; en un edificio, por el contrario, puede producir grandes daños.

\section{Disminución de $X_{2}$ por un trazado apropiado del cable:}

El cálculo del ejemplo demuestra que el valor decisivo para la disminución de la fuerza de pretensado es la hiperestática $\mathrm{X}_{2}$. La magnitud de $\mathrm{X}_{2}$ depende de $\delta_{20}$ y $\delta_{10}$, siendo $\delta_{20}$ diez veces mayor que $\delta_{10}$.
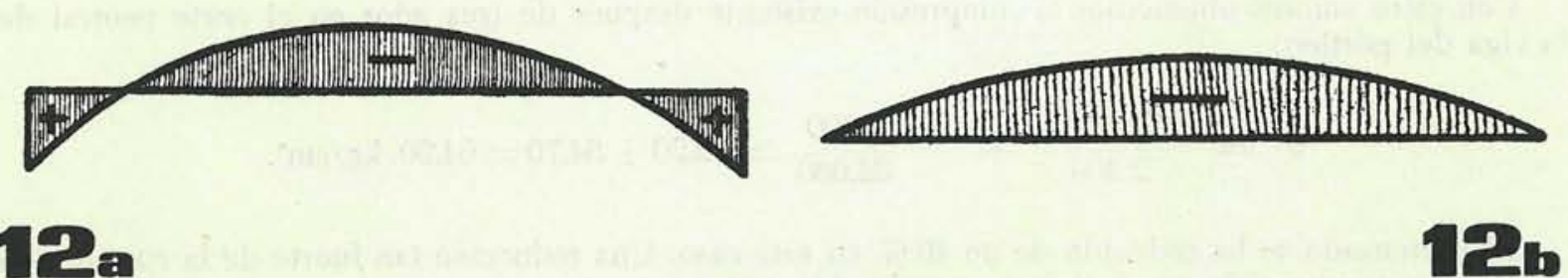

$\delta_{20}$ es el ángulo de apertura en la junta $n-n$, originado por el momento debido al pretensado, según se ha indicado en la figura 12a.

Por una modificación introducida en el trazado del cable podemos obtener un diagrama de $\mathrm{M}_{0}$, según la figura $12 \mathrm{~b}$.

Se deduce fácilmente que se puede reducir así la deformación $\delta_{20}$ hasta cero, lo cual conduce a una disminución considerable de la magnitud de $\mathbf{X}_{2}$. 